\title{
REVISIÓN DE LAS TÉCNICAS DE RECUPERACIÓN POST ENTRENAMIENTO MÁS USADAS PARA DISMINUIR LA INCIDENCIA DE FATIGA CRÓNICA EN FUTBOLISTAS
}

\author{
REVIEW OF THE MOST WIDELY USED POST-TRAINING RECOVERY \\ TECHNIQUES TO REDUCE THE INCIDENCE OF CHRONIC FATIGUE IN \\ FOOTBALL PLAYERS
}

\author{
Alexandrea Gaviria Marulanda ${ }^{1}$ \\ Luisa María Zapata Segura ${ }^{2}$ \\ Estefany Echeverry Mosquera ${ }^{3}$ \\ Mayra Alejandra Vásquez Vallejo ${ }^{4}$ \\ Ingrid Tatiana Alegría Riascos ${ }^{5}$ \\ Diana Carolina Ríos Ararat ${ }^{6}$ \\ Paola Andrea Pedroza Murillo ${ }^{7}$ \\ Aceptado: $30 / 11 / 2020$ \\ Publicado online: 14/12/2020
}

\begin{abstract}
RESUMEN
Introducción: El siguiente artículo pretende resaltar el uso de las técnicas de recuperación más utilizadas en los futbolistas posterior al entrenamiento y competencia deportiva, que disminuyan la incidencia de fatiga crónica y a su vez, la ocurrencia de lesiones osteomusculares, mejorando el rendimiento deportivo y evitando el síndrome de sobreentrenamiento por falta de recuperación. Objetivo: Indagar en los diferentes protocolos o estrategias de recuperación más utilizadas en el post entrenamiento en futbol y la labor del fisioterapeuta deportivo en los equipos multidisciplinarios. Métodos: Se revisaron bases de datos como Pubmed, Science direct, Scielo, Google Scholar, a través de una revisión exploratoria, generando un análisis frente a la efectividad e impacto a nivel fisiológico y psicológico. Resultados: Los resultados permitieron agrupar las técnicas de recuperación en el grupo de métodos de recuperación activa y el de métodos de recuperación pasiva que favorecen la recuperación del deportista tras las sesiones de entrenamiento y en periodos de competencia. Conclusión: Diferentes autores han señalado la relevancia que tiene la recuperación en el rendimiento deportivo y especialmente en este tipo de deporte, pues constituye uno de los principios básicos del entrenamiento, en la cual se pueden prevenir las lesiones en el futbol desde diferentes aspectos.
\end{abstract}

Palabras claves: Entrenamiento, Recuperación Deportiva, Fatiga Muscular, Competencia, Lesión Deportiva, Estrés Deportivo.

\footnotetext{
${ }^{1}$ Fisioterapeuta, Magister en prevención de riesgos laborales, Universidad Santiago de Cali-Colombia. alexagaviria60@gmail.com . (D) $0000-0003-0645-9675$

2 Fisioterapeuta, Universidad Santiago de Cali-Colombia. luisasegura1807@gmail.com . (i) 0000-0003-0457-7715

${ }^{3}$ Fisioterapeuta, Universidad Santiago de Cali-Colombia. stefyecheverry45@gmail.com. (D)0000-0003-1448-9612

${ }_{4}^{4}$ Fisioterapeuta, Universidad Santiago de Cali-Colombia. malejandravasquez0219@gmail.com. (D)0000-0001-8011-1170

${ }^{5}$ Fisioterapeuta, Universidad Santiago de Cali-Colombia. tatianaalegria81@gmail.com. (DD0000-0003-4668-0024

${ }^{6}$ Fisioterapeuta, Universidad Santiago de Cali-Colombia. dianacarolinariosararat@gmail.com. (D)0000-0003-1084-121X

${ }^{7}$ Fisioterapeuta, Universidad Santiago de Cali-Colombia. paola.pedroza00@usc.edu (iD0000-0002-7650-1747
} 


\begin{abstract}
Introduction: The following article aims to highlight the use of the most used recovery techniques in soccer players after training and sports competition, which reduce the incidence of chronic fatigue and in turn, the occurrence of musculoskeletal injuries, improving sports performance and avoiding overtraining syndrome due to lack of recovery. Objective: To investigate the different recovery protocols or strategies most used in soccer post-training and the work of the sports physiotherapist in multidisciplinary teams. Methods: Databases such as Pubmed, Science direct, Scielo, Google Scholar, were reviewed through an exploratory review, generating an analysis against the effectiveness and impact at a physiological and psychological level. Results: The results allowed to group the recovery techniques into the group of active recovery methods and that of passive recovery methods that favor the recovery of the athlete after training sessions and in competition periods. Conclusion: Different authors have pointed out the relevance of recovery in sports performance and especially in this type of sport, since it constitutes one of the basic principles of training, in which injuries in soccer can be prevented from different aspects.
\end{abstract}

Key words: Training, Sports Recovery, Muscle Fatigue, Competition, Sports Injury, Sports Stress.

\title{
SUMÁRIO
}

Introdução: O seguinte artigo tem como objetivo destacar a utilização das técnicas de recuperação mais utilizadas em jogadores de futebol após treinamento e competição esportiva, que reduzem a incidência de fadiga crônica e por sua vez, a ocorrência de lesões musculoesqueléticas, melhorando o desempenho esportivo e evitando síndrome de overtraining devido à falta de recuperação. Objetivo: Investigar os diferentes protocolos ou estratégias de recuperação mais utilizados no pós-treinamento do futebol e a atuação do fisioterapeuta esportivo em equipes multidisciplinares. Métodos: Bases de dados como Pubmed, Science direct, Scielo, Google Scholar foram revisadas por meio de uma revisão exploratória, gerando uma análise de efetividade e impacto em nível fisiológico e psicológico. Resultados: Os resultados obtidos permitiram agrupar as técnicas de recuperação no grupo dos métodos ativos de recuperação e nos passivos que favorecem a recuperação do atleta após os treinos e em períodos de competição. Conclusão: Diferentes autores têm apontado a relevância da recuperação no desempenho esportivo e principalmente neste tipo de esporte, uma vez que constitui um dos princípios básicos do treinamento, no qual as lesões no futebol podem ser prevenidas de diversos aspectos.

Palavras-chave: Treinamento, Recuperação Esportiva, Fadiga Muscular, Competição, Lesão Esportiva, Estresse Esportivo.

\section{INTRODUCCIÓN}

El fútbol es uno de los deportes con mayor influencia a nivel mundial, gracias a lo lucrativo que resulta para quienes se dedican a esta profesión y a la popularidad que tiene el deporte en todo el planeta, tanto en el ámbito administrativo como deportivo (Moreno, 2016).

Con el entrenamiento regular e intenso, algunos deportistas experimentan sobreentrenamiento, sobrecarga o fatiga crónica; si las cargas de trabajo no se modulan, el deportista puede incurrir en el riesgo de sufrir de síndrome de sobreentrenamiento (SSE), el cual se caracteriza por un descenso en el rendimiento que puede asociarse a alteraciones fisiológicas y psicológicas, necesitando en algunas ocasiones varias semanas o incluso meses de recuperación y como consecuencia, el deportista puede perder toda una temporada competitiva o llegar a abandonar incluso su carrera deportiva, acompañado en algunas ocasiones de lesiones musculares. 
Es importante resaltar que se requiere una adecuada relación trabajo-descanso, que faciliten los procesos regenerativos del organismo cuando se exceden las exigencias físicas individuales de regulación y adaptación, que puede generar afecciones a nivel muscular, cardiorrespiratorio, sistema nervioso, psicológico, metabólico y endocrino, presentando fatiga crónica, denominada también síndrome de sobreentrenamiento (Sandoval, 2003).

La fatiga puede clasificarse según la duración y el lugar de aparición (central o periférica). Según la duración, puede ser aguda, la cual aparece durante o posterior a una sesión de entrenamiento o competencia deportiva; subaguda, cuando el deportista presenta una sobrecarga secundaria a uno o varios microciclos relativamente intensos, con poca recuperación y acúmulo de fatiga residual; y crónica, cuando se presenta posterior a 4 semanas de actividad o en la combinación de dos mesociclos, con un cúmulo de carga residual y un desbalance entre el entrenamiento, la competencia y la recuperación (Sandoval, 2003).

Dentro de los síntomas a nivel psicológico, se puede presentar depresión, apatía, perturbaciones del estado de ánimo, hiperreactividad del estrés, incremento de la ansiedad, así como también, la disminución en la autoestima y dificultad para concentrarse (Ferran et al, 2003).

Existe un posible papel de los hidratos de carbono en el sobreentrenamiento; el agotamiento gradual de estas reservas corporales con el entrenamiento agotador repetitivo puede contribuir al síndrome de sobreentrenamiento. Se ha demostrado que con el sobreesfuerzo el glucógeno del músculo empieza a agotarse, aunque haya una alimentación entre 40 y $60 \%$ de calorías totales en forma de hidratos de carbono (William, 2007).

Por otro lado, tener una buena fase de recuperación permite que el corazón retome el ritmo cardiaco habitual, se normalice la temperatura corporal, se disminuya el riesgo de sufrir lesiones y ayuda a que el resto de los órganos retomen sus funciones con normalidad, favorece la recuperación muscular y evita la sobrecarga del musculo (William, 2007).

El fútbol es un deporte de mucho contacto, que expone a sus practicantes a sufrir lesiones de manera constante. Según un estudio realizado por (Belloch, Pérez \& Figueres, 2010); sobre la epidemiología en el fútbol, afirma que: La mayoría de las lesiones se registran en las extremidades inferiores, con una incidencia de lesiones de 2 a 9,4 por cada $1000 \mathrm{~h}$ de exposición. Predominan sobre todo los esguinces, seguidos de las fracturas, las distensiones musculares, rotura de ligamentos, afectaciones en el menisco y contusiones. Las lesiones de rodilla son las más comunes, seguidas por las lesiones de tobillo. La frecuencia de lesiones es mayor durante la competición que durante los entrenamientos (Ros, 2015).

Así mismo, las lesiones en las ligas de Colombia también son motivo de preocupación y alarma, ya que un estudio realizado por (Osorio et al, 2007) en la ciudad de Medellín, afirma que las lesiones deportivas más frecuentes son en rodilla, tobillo y pie, las cuales se presentan en deportes de impacto que implican saltar, golpear. La mayoría de los deportes se presentan con lesiones en miembros inferiores con un 55\% y un $90 \%$ con mayor frecuencia en la región de la cadera y el pie, con mayor predominio de esguinces, tensiones y contusiones. Estudios realizados sobre la aparición de lesiones demuestran una incidencia del $10 \%$ al $15 \%$ para el tobillo y del $3 \%$ al $15 \%$ para el pie (Belloch et al, 2010).

Frecuentemente los músculos se autolesionan al estirar o al realizar contracciones exageradas. Biomecánicamente este accidente ocurre en los músculos poliarticulares en donde se exige una tensión violenta y brusca, con asincronismo articular. Como es el caso del musculo recto anterior del cuádriceps y del sartorio en el chut de un futbolista: ambas acciones se ponen en función simultáneamente (flexión de cadera y extensión de esta) (Iturr, 2010).

Un mal entrenamiento, bien sea por defecto por exceso, un mal calentamiento y la fatiga muscular al final de una competencia hacen que se originen los accidentes musculares, tales como contusiones, contracturas, calambres y rupturas musculares (Iturr, 2010). 


\section{Análisis comparativo de las técnicas más utilizadas en la recuperación post entrenamiento en el futbol.}

Los medios de recuperación se clasifican en cuatro categorías de acuerdo con sus características, los medios de recuperación activa son una de ellas, pues se emplean elementos como el trote suave y el estiramiento muscular. Otro son los métodos fisioterapéuticos, donde están la masoterapia, electroterapia e hidroterapia, entre otros. Finalmente, encontramos los de recuperación pasiva, entre los que se cuentan el sueño y el descanso (Moreno, 2016).

Estos medios de recuperación son de gran importancia y también son mencionados por otros autores como estrategias de recuperación.

\section{Métodos de recuperación activa}

\section{Estiramientos y Resistencia aeróbica regenerativa}

Los estiramientos musculares de tipo estáticos pasivos relajados, son recomendados para recuperar la posición de reposos del músculo una vez finalizada la actividad; se recomienda mantener una posición de estiramiento de forma relajada durante un tiempo estimado de 30" y 1 minuto de manera sostenida; la tensión de elongación debe ser ligera y soportable, no dolorosa para el deportista. Hay otros estudios, por el contrario, que indican que es más efectivo realizar estiramientos pasivos dinámicos, ya que, tras la ejecución de este tipo de estiramientos, disminuye un $16 \%$ la rigidez muscular. Por otro lado, hay estudios que comprueban que el estiramiento estático aplicado inmediatamente tras el ejercicio de fuerza de alta intensidad genera un dolor muscular tras 48 horas (García, 2015).

La resistencia aeróbica regenerativa, es un mecanismo de recuperación biológica, que se utiliza como parte del entrenamiento a las 24 horas posteriores a una competencia o al día siguiente de un entrenamiento muy intenso; con una intensidad de 60-70\% FCMax., con una duración de 20-30 minutos (Sandoval, 2003)

La recuperación activa es uno de los protocolos más usados en la recuperación de deportistas. Una carga de $50-60 \%$ del consumo máximo de oxígeno tras un esfuerzo intenso y con acúmulo importante de lactato, es capaz de aclarar ese lactato en sangre en los deportistas. Favorece a la recuperación mediante el incremento del flujo sanguíneo y de la acción de bombeo generada por la contracción-relajación de los músculos activos que acabará ayudando a la eliminación de sustancias de desecho, lo que puede incrementar o mejorar la reposición de sustratos energéticos haciendo llegar más sangre a las fibras musculares (García, 2015).

Dentro de las técnicas de recuperación empleadas se encuentra:

Carrera continua - Estiramientos (CET) C Consistió en realizar 8 minutos de carrera continua a una con frecuencias máximas teóricas entre el $60 \%$ y $70 \%$, que se calculó utilizando la ecuación de Tanaka 2001 (FCMT $=208,75-0,73^{*}$ Edad) y 8 minutos de estiramientos estáticos, realizando repeticiones de 15 segundos en la musculatura de gemelos, aductores, cuádriceps, glúteos, musculatura lumbar e isquiotibiales.

Carrera continua - Elevación piernas (CEL). Consistió en realizar 8 minutos de carrera continua a la intensidad descrita anteriormente y 8 minutos de elevación de piernas en supino, con flexión de caderas y rodillas formando un ángulo de 90 ㅇ.

Estiramientos - Elevación piernas (EE). Esta estrategia combinó 8 minutos de estiramientos estáticos como se ha descrito en CET y 8 minutos de elevación de piernas siguiendo la metodología de CEL.

Recuperación control (RC). La recuperación rutinaria que realizaba el equipo durante la temporada se tomó como control por la imposibilidad de realizar recuperación pasiva (sentados 16 minutos) por indicaciones del cuerpo técnico. Es una ER simple (no combinada) de una duración total de 5 
minutos, la cual consistió en la realización de 20 segundos de estiramientos de los siguientes grupos musculares gemelos (García, 2015).

\section{Masoterapia}

El masaje en un efecto muscular produce una disminución en la excitabilidad de la motoneurona alfa lo que llevará a aliviar los espasmos musculares y a interrumpir el ciclo dolor-tensión-dolor, y a su vez aumentará el flujo sanguíneo del músculo y así mejorar el tono muscular, esto hará que el deportista pueda sentir una relajación y una calma en su cuerpo (Nereyda, 2016).

\section{Electroterapia}

La electroestimulación está basada en la transmisión de impulsos eléctricos a través de electrodos superficiales situados en la piel que estimulan las motoneuronas a nivel periférico llevando a la contracción muscular, justificándose su uso para la recuperación en que esas contracciones pueden ser ventajosas por incrementar el flujo sanguíneo mediante un efecto de "bombeo", lo que ayudaría a la reparación muscular (García, 2015).

\section{Hidroterapia}

Los beneficios del agua combinada con el efecto térmico, aporta a los efectos de recuperación de actividades deportivas pues que esta técnica facilita la reducción del edema muscular y el incremento de la respuesta cardiaca (sin incremento del gasto energético), aumenta el flujo sanguíneo y el transporte de nutrientes, así como la eliminación de productos de desecho (García, 2015).

\section{Crioterapia}

La crioterapia desde un efecto fisiológico lleva a la contracción de los vasos sanguíneos, y reduce la velocidad de la conducción nerviosa, lo que va a disminuir el espasmo y el tono muscular, esta técnica es administrada de diferentes formas como bolsas de hielo triturado de manera directa en la zona a tratar, masaje con hielo: movimientos de frotación. Compresas frías: compresas de gel congeladas para aplicación directa, inmersión: introducir el área a tratar en un recipiente con hielos y agua, y aerosol o spray: se utiliza cloruro de etilo, aplicado directamente en la zona a tratar (Rodríguez, 2018).

\section{Métodos de recuperación pasiva}

\section{El descanso y el sueño}

Es importante mencionar que dentro del proceso de recuperación pasiva post entrenamiento, el descanso es muy importante puesto que, el sueño reparador es fundamental para los deportistas de élite, dado que no solo depende su desempeño físico sino mental. Al entrar en estado de reposo, el cuerpo reduce su frecuencia cardiaca, generando así mismo la secreción de la hormona del crecimiento $(\mathrm{GH})$, cuya función es fundamental a la hora de reparar y regenerar el daño muscular (García, 2019).

Los factores limitantes de una buena reparación en el foco son: el aporte de oxígeno, el nivel de vascularización y el aporte metabólico. Además de lo anteriormente mencionado, existen dos respuestas a nivel genético que condiciona la respuesta del tejido de forma individual, éstas son la respuesta epigenética, término acuñado por (Conrad Hal Waddington, 1942) para referirse al estudio de las interacciones entre genes y ambiente que se producen en los organismos, y por tanto, la epigenética es el conjunto de reacciones químicas y demás procesos que modifican la actividad del ADN pero sin alterar su secuencia; y la respuesta genómica, que podemos considerarla como aquellos mecanismos por los que el genoma responde a un estímulo externo o interno produciendo una activación/represión de genes y una modificación de la síntesis de proteínas (Tomás et,al, 2016). 


\section{Nutrición y ayuda Ergogénica}

Es importante determinar los requerimientos nutricionales de manera individual en los deportistas, propendiendo a una alimentación saludable y balanceada, con una adecuada distribución calórica, que garantice una adecuada reserva de glucógeno, los cuales dependen directamente de las características antropométricas del deportista como la edad, nivel competitivo, etapa de entrenamiento y competitiva, posición de juego, entre otras (Sandoval, 2003). Se recomienda adicionalmente, utilizar suplementos dietarios nutricionales con prescripción individual, con evidencia científica y saludable, no ingerir sustancias prohibidas y algunos estudios demuestran la efectividad del uso del plasma rico en plaquetas (PRP), denominado factores de crecimiento (Barcelona, 2009).

\section{DISCUSIÓN}

El masaje es una de las técnicas fisioterapéuticas de mayor arraigo dentro del fútbol de rendimiento. No obstante, a pesar de formar parte integral de los protocolos de recuperación, es prácticamente nula la evidencia científica específica de esta técnica en nuestro deporte, justificando su empleo en la creencia de sus efectos, biomecánicos, fisiológicos, neurológicos y psicológicos sobre el edema y el dolor, un incremento de la eliminación del lactato (Rico, et al, 2018)

Sin embargo, dentro del masaje las técnicas como el amasamiento superficial y profundo, en ocasiones con bolsas de hielo, es una estrategia válida para la recuperación intercesión en el fútbol, a pesar del arraigo, anteriormente anotado, dentro del ámbito de la fisioterapia en el fútbol, sigue sin existir evidencia empírica concluyente que apruebe la utilización de este medio regenerador tras la competición (Fernadíz, 2005).

Las técnicas de recuperación con varias formas de inmersión en agua se han vuelto muy populares en los atletas elite. Las formas más comunes de inmersión en agua son la inmersión en agua fría (IAF), inmersión en agua caliente (IAC) y la terapia de contraste de agua (TCA), en la cual los atletas alternan entre la inmersión en agua caliente y fría (Dobarro et al, 2019).

Por su parte Ascensão, Leite \& Rebelo (2017) indican que es muy importante la temperatura del agua a la hora de realizar la recuperación ya que estas pueden ser usadas como estrategia regeneradora: crioterapia (inmersión en agua fría, $\leq 15^{\circ} \mathrm{C}$ ), termoterapia (inmersión en agua caliente, $>36^{\circ} \mathrm{C}$ ) o baño de contraste (terapia con contraste en temperatura del agua).

A pesar de ser una técnica con mayor evidencia científica Chen, Miladi, Temfemo, Mandengué, \& Ahmaidi, en su estudio observaron que la hidroterapia representa el $58.3 \%$ de utilidad y a su vez genera unas respuestas fisiológicas favorables para la recuperación física como son los cambios en los fluidos intracelulares e intravasculares, aumentando el flujo sanguíneo y con ello el de nutrientes como la glucosa y proteínas, sumado a que se reducen tanto el $\mathrm{CO} 2$ como el ácido láctico, avasallando los edemas que se generan pots ejercicio (Moreno, 2016).

Otra técnica muy utilizada son los estiramientos post entrenamiento "los ejercicios de estiramiento relajan el músculo y disminuyen la acumulación del ácido láctico, favoreciendo la recuperación física" Chen, Miladi, Temfemo, Mandengué, \& Ahmaidi, quienes argumentan que la recuperación activa, dentro de las cuales se incluye el estiramiento, es una técnica muy utilizada en los procesos de recuperación física particularmente en los deportes, por lo cual se sigue investigando su efectividad (Moreno, 2016).

Pero también se evidencia que otros autores contradicen los beneficios del estiramiento tras el ejercicio, Torres, Ribeiro, Duarte y Cabri (2012), quienes después de realizar una revisión sistemática 
y metaanálisis, concluyeron que el estiramiento no tiene mayores soportes científicos sobre los efectos en la recuperación y regeneración muscular postejercicio.

Tradicionalmente, la crioterapia ha sido ampliamente utilizada después del entrenamiento, aunque existe cierta controversia científica relativa a su eficacia. Los resultados de este estudio demuestran que ayuda a la recuperación después del ejercicio físico, en nuestro caso concreto, percibiendo los jugadores una menor sensación de fatiga en el entrenamiento. Aunque anecdóticamente el estiramiento es una de las estrategias de recuperación más utilizadas, son escasas las publicaciones científicas que examinan los efectos del estiramiento como método de recuperación post-esfuerzo (González, 2017).

Sobre la electroterapia, (Zarzuel, 2016) en su tesis sobre métodos de recuperación en fútbol menciona: "En resumen, en los pocos estudios realizados hasta el momento, la EMS no mejoró la recuperación de forma significativa, aunque podría ser útil como medio de recuperación ya que no se obtienen desventajas en su utilización y se podría obtener una cierta ventaja sobre la recuperación pasiva. De igual forma un estudio de la eficacia de diferentes técnicas de recuperación post-ejercicio de (García, 2015) se puede encontrar que la electroterapia, siendo esta una técnica que dependiendo del modo en que aplique puede producir relajación muscular, o activación de estos.

Este hecho también se describe en otro estudio donde la electroestimulación no resultó ser más eficaz que la recuperación pasiva, en este caso en la recuperación de la contracción voluntaria máxima de los extensores de rodillas. Existen estudios que muestran cómo la estimulación de baja frecuencia no tiene efecto sobre el dolor muscular tras ejercicio, otros que muestran que tiene leves efectos positivos o bien se encuentran mejoras significativas en la sensación de dolor muscular y en la percepción de la recuperación. Este alivio del dolor muscular podría estar basado en una inhibición sensorial de fibras aferentes o en la facilitación de liberación de endorfinas, siendo el primero de estos hechos el más comprobado (García, 2015).

En cuento al descaso, para los atletas de cualquier deporte, el sueño es de vital importancia para la recuperación; se puede definir como El sueño podría ser definido como un estado de reposo físico y mental en el que una persona atraviesa por un estado relativamente inactivo e inconsciente asociado a procesos de recuperación, interviniendo en numerosas funciones biológicas. La necesidad de descanso de esta población es más grande que la del humano común. Una falta de sueño ya sea leve puede afectar de manera negativa considerablemente al organismo, en el mismo artículo Sánchez menciona El rendimiento deportivo se ve deteriorado incluso con pérdidas leves del sueño (4-5 h comparado con 7-8 h) observándose deterioros en velocidad, resistencia y precisión; por otro lado se encuentran efectos positivos sobre el sistema, algunos estudios han demostrado que la privación de sueño puede tener efectos importantes sobre la función inmune, los niveles de citocinas y de los marcadores de inflamación, disminuyendo también la función de las células NK, los linfocitos T y los monocitos (Moreno, 2012).

Es importante considerar entonces, diferentes aspectos a tener en cuenta para evitar la aparición de fatiga crónica, dentro de los cuales se destaca: Aplicar las diferentes técnicas de recuperación post entrenamiento y periodos de competencia, tener una adecuada relación trabajo/descanso posterior al entrenamiento deportivo y periodo competitivo, detección temprana y reconocimiento individual de los síntomas que puedan indicar presencia de fatiga en el deportista principio de individualización del entrenamiento, control médico y de laboratorio que permitan una valoración funcional eficiente del atleta, correctos hábitos de alimentación, considerando el volumen indicado de calorías de manera individual, identificar claramente los indicadores de salud del deportista y de estrés emocional, utilizar una adecuada hidratación, reposición hidromineral y energética durante el entrenamiento y la competencia, disponer de indumentaria deportiva adecuada, con mayor 
importancia en el calzado, que permita desarrollar correctamente el centro de gravedad y poder así evitar lesiones deportivas.

El consumo de complementos nutricionales por parte de los deportistas de élite en la actualidad es un hecho inocultable, al tener elevadas demandas energéticas que implican un esfuerzo físico permanente y extenuante; sumado a la regeneración permanente de tejido que tienen que hacer los diversos sistemas orgánicos implicados en la actividad deportiva, los cuales requieren de una alta dosis de nutrientes, que generalmente no alcanzan a ser suplidos con la dieta cotidiana (Moreno, 2012).

En consecuencia, a una mala preparación o recuperación aumenta la incidencia de que el deportista sufra lesiones, por esta razón, desde un concepto fisioterapéutico, se debe hacer énfasis en que los trabajos de prevención de lesiones deben estar caracterizados por habilidades específicas del componente motor y sensorial del movimiento corporal humano, con el fin de potencializar cualidades físicas del deportista. Por ejemplo, existen habilidades paramétricas, que son base para el desarrollo del potencial del futbolista, a saber: fuerza, velocidad, resistencia y flexibilidad. Después, se encuentran las habilidades sinergísticas, que se basan en actividades de co-contracción y activación recíproca. Por último, encontramos las habilidades compuestas, que son una combinación de dos, o más habilidades que dan como resultado balance, equilibrio, coordinación, relajación motora, salto y carrera, las cuales son gestos funcionales en el fútbol. Adicionalmente, se encuentran las habilidades de interacción sensorial de los sistemas propioceptivo, visual, auditivo y somatosensorial, que desarrollan en su totalidad el potencial del movimiento corporal humano (Mantilla, 2018).

\section{CONCLUSIÓN}

Se ha logrado identificar, que es de vital importancia el uso de técnicas de recuperación post entrenamiento, así como también la correcta planificación de las sesiones de entrenamiento y periodos de competencia para evitar así la fatiga crónica o síndrome de sobreentrenamiento; a su vez, es importante realizar controles de campo y de laboratorio de manera periódica, que permitan realizar una detección temprana de cualquier síntoma asociado a este tipo de alteraciones.

Se debe propender el trabajo de manera multidisciplinaria en el abordaje del deportista, siendo un equipo colectivo ideal entre entrenadores, médicos, psicólogo, nutricionista y fisioterapeuta, que puedan planificar y establecer controles oportunos en la incidencia de este tipo de complicaciones, teniendo en cuenta el rendimiento del deportista durante el entrenamiento y competencia y su respuesta biológica.

El uso de técnicas post entrenamiento, se clasificaron en métodos de recuperación activa y métodos de recuperación pasiva.

Dentro de los métodos de recuperación activa, se resaltó el uso de las técnicas más utilizadas, las cuales fueron: el estiramiento muscular, la resistencia aeróbica regenerativa, la masoterapia, la electroterapia, la hidroterapia y la crioterapia; y dentro de los métodos de recuperación pasiva, se destacaron los beneficios del descanso y sueño, y la importancia de una adecuada nutrición y uso de suplementos dietarios prescritos por el médico tratante o nutricionista.

En consecuencia, mientras los planes de recuperación física no varíen, todo dependerá del proceso que decida tomar el fisioterapeuta: ejercicios de recuperación activa, recuperación pasiva, evaluación constante de la evolución de la lesión, variaciones en el plan de trabajo, entre otras (ejercicios adecuados para cada lesión, teniendo en cuenta los factores morfológicos y extrínsecos del individuo, así como las sesiones de rutina) para lograr una evolución constante y segura de la lesión; pero también dependerá del compromiso que tenga el propio deportista con el proceso que debe llevar; de tal manera que se logre la evolución, rehabilitación y recuperación deseada durante el proceso que el profesional en salud decida tomar. 


\section{REFERENCIAS BIBLIOGRÁFICAS}

Dobarro Magan, D. (2019). Eficacia de la recuperación activa en medio acuático para la fatiga post-partido en futbolistas aficionados. Diseño cruzado aleatorizado. Recuperado el 18 de 05 de 2020, de UCJC: https://repositorio.ucjc.edu/handle/20.500.12020/864

Ferran Suay, J. R. (04 de 2003). Indicadores psicologicos de sobre entrenamiento y agotamiento. Psicologia del deporte, 9(13).

Ferrandiz, S. (1995) Quiromasaje: técnica y sensibilidad. Recuperado el 18 de 05 de 2020, de http://www.sld.cu/galerias/pdf/sitios/rehabilitacion-

bio/fisioterapia_quiromasaje_tecnica_y_sensibilidad.pdf

Garcia Concepción, M., Peinado, A., Paredes Hernandez, V., \& Alvero-Cruz, J. (06 de 2015). Eficacia de diferentes estrategias de recuperación en jugadores de futbol de elite. Revista internacional de medicina y ciencias de la actividad fisica del deporte(58).

García de la Concepción, M. Á. (2015). Estudio de la eficacia de diferentes técnicas de recuperación postejercicio. Recuperado el 19 de 05 de 2020, de Riuma: https://riuma.uma.es/xmlui/bitstream/handle/10630/11631/TD_Garcia_de_la_Concepcion_Miguel _Angel.pdf?sequence $=3$ \&isAllowed $=y$

Garcia, E. (2019). Recuperación en deportistas aplicación de la electroestimulación por el terapeuta Recuperado el 18 de 05 de 2020, de Docplayer: https://docplayer.es/169723194-Autor-enriquegarcia-martin.html

González, O. d. Métodos de recuperación en futbolistas: crioterapia y estiramientos activos Recovery methods on football players: cryotherapy and active stretching (2017). Recuperado el 18 de 05 de 2020,

https://repositorio.unican.es/xmlui/bitstream/handle/10902/5820/RED\%20GONZ\%c3\%81LEZ,\%20

Oihana\%20de\%20la.pdf?sequence=1\&isAllowed=y

Iturr, J. J. (2010). Lesiones musculares y deporte. Scielo, 4(2).

Osorio Ciro, M. P. (6 de 2007). Lesiones deportivas. Revista iatreia, 20(2).

Llana Belloch, S., \& Pérez Soriano, P. y. (01 de 2010). La epidemiología en el fútbol: una revisión sistemática. Revista Ef deportes, 10(37).

Mantilla, J. I. (2018). Propuesta de un protocolo de prevención de lesiones deportivas en futbolistas profesionales, basado en una revisión sistematica de la literatura. Revista de educación fisica, 7(4).

Moreno Bolivar, H., \& Ramos Bermudez, S. a. (2012). Recovery media an interdisciplinary work in the coaches attending thesouth American Games Medellín 2010 (Colombia). Rev. Salud Uninorte, Scielo.org.co., 29(1).

Moreno, H., Ayala, C. F., \& Melo, L. G. (09 de 2016). Caribe medios de recuperación utilizados por entrenadores en los IV juegos intercolegiados centroamericanos y del caribe. Preparación fisica(4).

Nereyda, A. R. (2016). Influencia del masaje con crioterapia (znar) sobre la recuperación posterior a una competencia en corredores de fondo Recuperado el 18 de 05 de 2020, de http://eprints.uanl.mx/12820/

Rico-RosilloMG, V.-R. G. (2018). Sueño y sistema inmune. Rev Alerg Mex, 65(2).

Rodriguez, B. (2018). Análisis de la fatiga y la recuperación en el baloncesto en relación con la efectividad de tiro Estudio experimental en jugadores de diferente nivel Recuperado el 18 de 05 de 2020, de http://ddfv.ufv.es/bitstream/handle/10641/1493/Antonio_Sastre.pdf?sequence=1

Ros, A. (09 de 2015). Metodos de recuperación post- esfuerzo en deportes. Revista Ef deportes. 
Sandoval, A. E. (2003). Diagnóstico y prevención de la fatiga cronica o del sindrome de sobreentrenamiento en el deporte de alto rendimiento. Una propuesta de mecanismos de recuperación biologica. Cuadernos de psicologia del deporte, 3(1).

Servicios médicos del futbol club Barcelona. (02 de 2009). Guia de practica clinica de las lesiones musculares. Epidemiologia, diagnostico, tratamiento y prevención. APUNTS MED ESPORT, 4.5.

Tomás F. Fernández Jaén, P. G. (09 de 2016). Criterios para el retorno al deporte después de una lesión. Archivos de medicina del deporte, 1(34).

William McArdle, F. K. (s.f.). Fundamentos de fisiología del ejercicio. En F. K. William McArdle, Fundamentos de fisiologia del ejercicio (pág. 37). Mc Graw Hill. 